Molecular tunnel junctions

DOI: $10.1002 / \mathrm{smll} .200600600$

\section{Protein Conduction and Negative Differential Resistance in Large-Scale Nanojunction Arrays**}

\author{
Giuseppe Maruccio, * Pasquale Marzo, \\ Roman Krahne, Adriana Passaseo, Roberto Cingolani, \\ and Ross Rinaldi
}

Molecular electronics ${ }^{[1]}$ is aimed at demonstrating suitable alternatives to silicon-based nanoelectronics for building complex and functional devices. One of the end goals is to use single molecules as active elements in electronic nanodevices, whereas the main challenges are the interconnection of the molecules and the fabrication of electrical contacts to the outside. In recent years, proteins have attracted much interest as active materials because of their electrontransfer (ET) capabilities (crucial for a number of vital processes such as photosynthesis, respiration, and energy conversion). These pivotal reactions usually take place inside a single protein (intramolecular ET) or between two or a few proteins (intermolecular ET). Therefore, it is crucial to develop reliable methods to interconnect and probe transport in single/few proteins in order to investigate the basic mechanisms underlying ET. This kind of information is not directly accessible by conventional techniques such as spectroscopic and electrochemical methods, which provide average figures over statistical ensembles. Up to now, such studies could only be performed by means of scanning probe methods (SPMs) ${ }^{[2-4]}$ but never in device geometry, due to severe difficulties in the fabrication of devices with single molecules as active components. The physical limitations of optical lithography have prompted the development of alternative, top-down techniques for patterning below the $100-\mathrm{nm}$ scale, but in most cases these do not match the advantages of photolithography in its low cost and high throughput (with the notable exception of nanoimprint lithography ${ }^{[5]}$ ). Most of these alternative methods (including mechanical break junctions, electron-beam lithography, electromigration, electrodeposition, etc.) are appropriate for contacting only single devices. Thus, despite the proof-of-concept for

[*] Dr. G. Maruccio, P. Marzo, Dr. R. Krahne, Dr. A. Passaseo, Prof. R. Cingolani, Prof. R. Rinaldi National Nanotechnology Laboratory of CNR-INFM Unità di Ricerca IIT, Distretto Tecnologico ISUFI Via Arnesano, 73100 Lecce (Italy) Fax: $(+39)$ 0832-298-180 E-mail: giuseppe.maruccio@unile.it

[**] The authors gratefully acknowledge the support of the SAMBA and the SPIDME European projects, the IIT and the Italian MIUR (FIRB Molecular Nanodevices). Moreover, we thank Franco Calabi, Ray Phaneuf, Marco Zanella, Teresa Todaro, and Vittorianna Tasco for fruitful discussions and the providing of materials, and Eliana D'Amone for technical assistance. individual components, ${ }^{[6-11]}$ the economical fabrication of more complex molecular circuits on a large scale remains a challenge.

Recently, Krahne et al. ${ }^{[12]}$ have succeeded in the fabrication of a network of nanojunctions defined only by optical lithography and wet etching of an $\mathrm{AlGaAs} / \mathrm{GaAs}$ quantumwell (QW) structure (an important step towards low-cost mass production). The gap size is determined by the thickness of the quantum well and of the deposited metal layer with subnanometer precision. This approach enabled the authors to investigate transport through single Au nanoclusters at $T=4 \mathrm{~K}$. However, the main drawback of this technique lies in the relatively large bulk leakage currents through the semiconductor substrate at ambient conditions, which limit the use of the nanojunctions to cryogenic temperatures in the dark and highly conductive nano-objects. In this Communication, we report on i) a significant improvement of this method and ii) the fabrication and characterization of a new class of nanodevices based on few/single proteins that operate at room temperature. Transport in molecular tunnel junctions based on the blue-copper protein Azurin is investigated for the first time at the single-molecule level in device geometry. We observe features characteristic of transport through molecules, such as negative differential resistance (NDR) in the current-voltage characteristics and identify the mechanism and the protein sites responsible (despite recent reports based on SPMs, the NDR phenomenon is little understood on the molecular scale ${ }^{[13]}$ ).

The nanojunction fabrication started with an AlGaAs/ GaAs (20 nm thick) quantum-well structure grown by metal-organic chemical vapor deposition (MOCVD) (Al concentration between $35 \%$ to $90 \%$ ). Mesa structures were defined by optical lithography and wet etching. Next, selective wet etching (using citric acid) was employed to remove a few tens of nanometers of the GaAs layer from the sloped edges of the mesa structure (Figure $1 \mathrm{a}$ ). To reduce bulk leakage currents, we employed a selective oxidation technique, carried out after the selective etching and the removal of the GaAs cap layer, which allows the conversion of the Al-rich layers into a stable native oxide (Figure 1b). This relies on a continuous flow of water steam carried by nitrogen gas that bubbles through deionized water. The oxidation is carried out at a controlled temperature in a home-made oven. After oxidation, the electrodes were defined by optical lithography and evaporation of a 15-nm-thick film of Ti/ $\mathrm{Pt}$. The gap is determined by: 1) the thickness of the GaAs QW, 2) the thickness of the deposited metal layer, and 3 ) the surface roughness of the etched $\mathrm{AlGaAs} / \mathrm{GaAs}$ interface. Figure $1 \mathrm{c}$ shows that the resulting gap between electrodes is only a few nanometers across; no significant changes in interface roughness are observed after oxidation. In such nanogaps, molecules/nanoparticles can be positioned between the electrodes by electrostatic trapping or by specific immobilization procedures. Since only photolithography is used to define the electrode pattern, the improved mesa-gap technique reported here enables the simultaneous, economic fabrication of large arrays of nanodevices working at ambient conditions. We emphasize that all the described processes are carried out at wafer scale, which is crucial for 


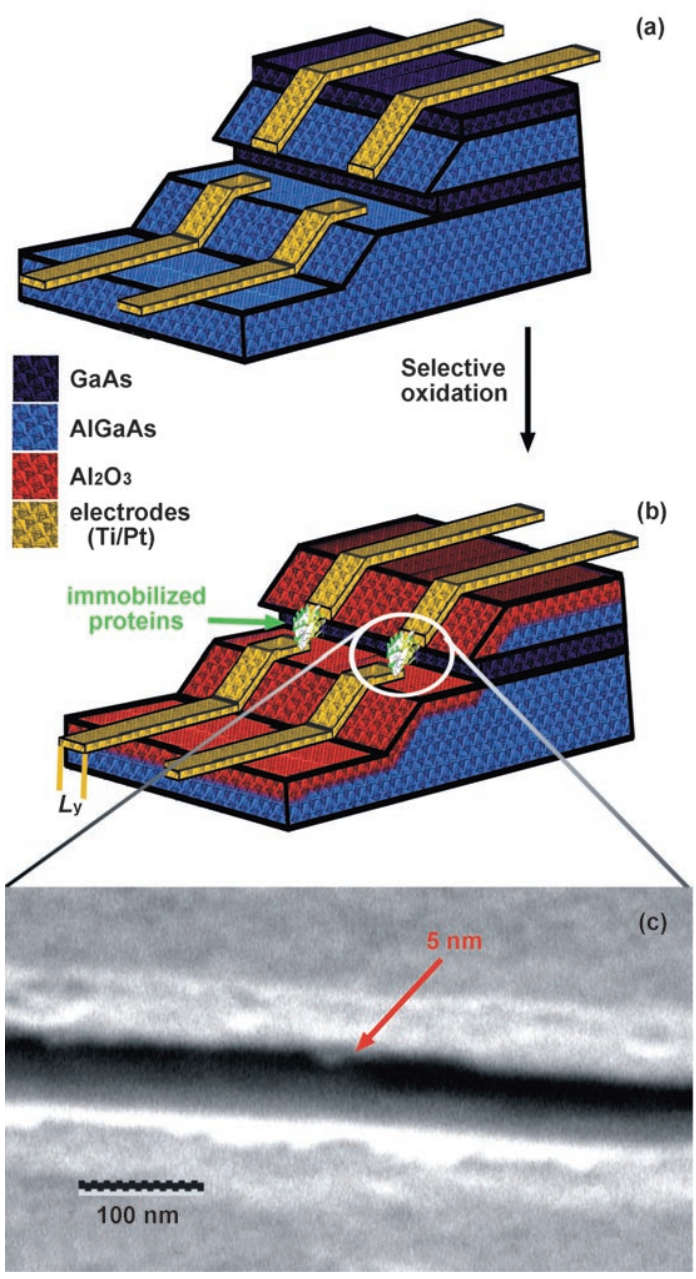

Figure 1. Schematics of the a) non-oxidized and b) oxidized mesa nanojunctions with immobilized proteins, and c) scanning electron microscopy image of a nanojunction with a 5-nm gap (see the restriction indicated by the arrow) fabricated on an oxidized sample. The etched groove separating the electrodes is visible in the horizontal direction. Due to the corrugated profile of the electrodes characterized by the presence of a few restrictions, few proteins in parallel participate in the current transport; only those immobilized in positions such as the one indicated by the arrow where the electrode distance matches the Azurin diameter (about 4-5 nm) are able to participate.

the implementation of low-cost mass-production of nanoscale devices.

Figure $2 \mathrm{a}$ and $\mathrm{b}$ shows the leakage current of a series of devices with high $\mathrm{Al}$ concentration $(80 \%)$ versus the oxidation time and oxidation temperature at a fixed source-drain voltage of $1.5 \mathrm{~V}$. We obtained as best parameters an oxidation time of $2 \mathrm{~h}$ at $450{ }^{\circ} \mathrm{C}$. We find for all conditions an almost exponential rise in leakage current with respect to the bias voltage (Figure $2 \mathrm{c}$ ), with values always below $30 \mathrm{pA}$ for highly oxidized samples (i.e., a reduction of six orders of magnitude with respect to the non-oxidized devices). Lower $\mathrm{Al}$ concentrations result in higher currents, because of a reduction of the oxidation rate.

Transport was investigated in Azurin, a small bluecopper protein $(14.6 \mathrm{kDa})$ from the bacteria P. aeruginosa,

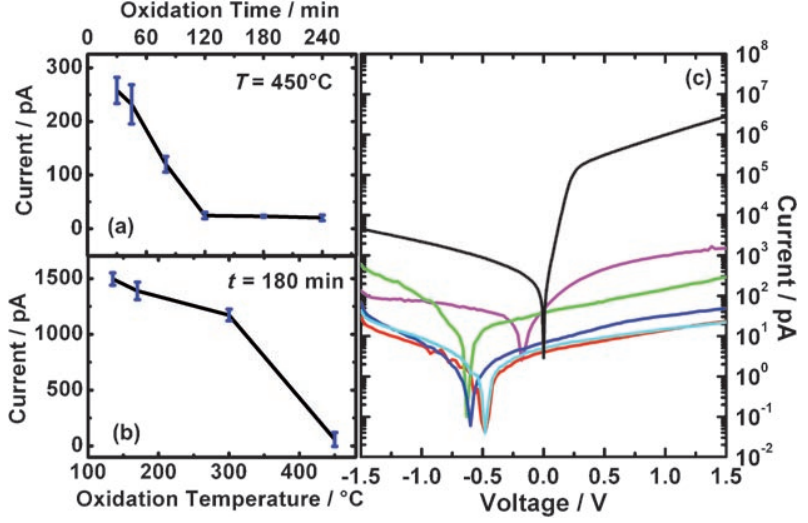

Figure 2. $\mathrm{a}, \mathrm{b}$ ) Dependence of the leakage current (at $V_{\text {bias }}=1.5 \mathrm{~V}$ ) on the oxidation temperature and duration. The Al concentration was kept constant at $80 \%$ for all these samples. The error bar shows the standard deviation from the final value of the leakage current in the oxidized samples. For optimal conditions, the leakage current remains below $30 \mathrm{pA}$ up to $V_{\text {bias }}=1.5 \mathrm{~V}$. c) $I-V$ characteristics of differently oxidized nanojunctions at room temperature and in the dark. Black line: no oxidation; green: $30 \mathrm{~min}, 450^{\circ} \mathrm{C}$; dark blue: $60 \mathrm{~min}$, $450^{\circ} \mathrm{C}$; red: $180 \mathrm{~min}, 450^{\circ} \mathrm{C}$; light blue: $240 \mathrm{~min}, 450^{\circ} \mathrm{C}$; pink: $180 \min , 135^{\circ} \mathrm{C}$.

which is able to mediate electron transfer in vitro from cytochrome $c_{551}$ to the nitrite reductase from the same organism. ${ }^{[14]}$ There is considerable interest in this protein that has recently been used to demonstrate biosensor devices ${ }^{[15]}$ and the first protein transistors. ${ }^{[1]}$ These devices exploit the charge transfer through the redox level of its copper site. Azurin exists in two stable electronic configurations $\mathrm{Cu}^{\mathrm{I}}$ and $\mathrm{Cu}^{\mathrm{II}}$, and its ET capability depends on the equilibrium between these two oxidation states by means of a reversible redox reaction, which continuously converts the $\mathrm{Cu}^{\mathrm{II}}$ copper oxidized state into the $\mathrm{Cu}^{\mathrm{I}}$ reduced state and vice versa.

In the work reported here, we immobilized wild-type Azurin molecules by direct site-specific attachment via the disulfide bridge (RSSR) onto our oxidized mesa nanojunctions. In detail, the mesa devices were functionalized with Azurin by casting of a $20 \mu \mathrm{L}$ drop of the protein solution (1.0 $\mathrm{mg} \mathrm{mL}^{-1}$ in $50 \mathrm{~mm} \mathrm{NH}_{4} \mathrm{Ac}$ buffer, $\mathrm{pH} 4.6$ ). After incubation (30 min at room temperature), the buffer solution was removed and the samples were washed, and then dried by a high-purity nitrogen flow. Since a single disulfide-bond site is present in Azurin, the protein orientation is defined once attached to the electrode (with the direction from the disulfide to the $\mathrm{Cu}$ site perpendicular to the electrode surface ${ }^{[16]}$ ). The width $L_{\mathrm{y}}$ of the electrodes, defined by optical lithography, was set at either 2 or $4 \mu \mathrm{m}$. However, the profile of the electrodes (see Figure 1c) is quite corrugated and only in a few restricted situations does the electrode distance match the Azurin diameter (about 4-5 nm). As a consequence, only a few proteins in parallel participate in the current transport: those immobilized in positions like the one indicated by the arrow in Figure $1 \mathrm{c}$. Therefore, intramolecular ET in a single or few Azurin molecules in parallel is probed here for the first time at the molecular level in device geometry (in our previous report, the electrode gap was $100 \mathrm{~nm}$ 


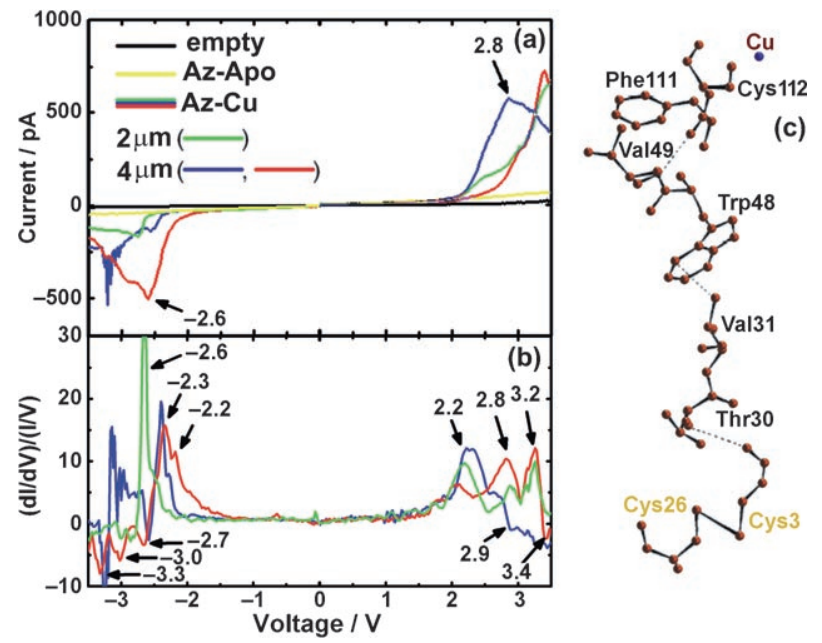

Figure 3. a) $I-V$ characteristics of various mesa devices functionalized with Azurin by casting of a $20 \mu \mathrm{L}$ drop of protein solution. The peaks in the current can be ascribed to resonant tunneling between localized states on the disulfide bridge RSSR and the copper site $\mathrm{Cu}^{\prime \prime}$. The black and yellow lines show the current across an empty nanojunction and a device based on Apo-Azurin, respectively, as references. Molecular features (such as NDR) gradually disappear after a couple of weeks, in agreement with our previous experiments on the ageing of Azurin monolayers. ${ }^{[18]}$ b) Normalized conductance plotted as a function of bias voltage. c) Electron-transfer pathway from RSSR to $\mathrm{Cu}^{\text {"I }}$ in $P$. aeruginosa Azurin. ${ }^{[30]}$

and electron transport occurred by hopping from one reduced protein to an adjacent oxidized one $\left.{ }^{[11]}\right)$.

Figure 3 a shows current-voltage characteristics for some of the devices, where Azurin was immobilized in between $\mathrm{Ti} / \mathrm{Pt}$ electrodes, with a minimum gap size of about $5 \mathrm{~nm}$ (Figure 1c). The dominant features are maxima in the current (indicated by the arrows in Figure $3 \mathrm{a}$ ) that we observe in all samples in the range of $-3.5 \mathrm{~V}$ to $-2.5 \mathrm{~V}$ and $2.5 \mathrm{~V}$ to $3.5 \mathrm{~V}$, with some fluctuations in the peak position within different samples. ${ }^{[17]}$ Such maxima in the current correspond to NDR and an increased transparency of the molecular tunnel junction under appropriate biasing conditions. We remark that molecular features (such as NDR) gradually disappear after a couple of weeks, in agreement with our previous results on ageing of Azurin monolayers. ${ }^{[18]}$ The $I-V$ characteristics of the empty nanojunction and of a device based on the Apo-Azurin (i.e., the Azurin protein without the $\mathrm{Cu}$ atom) are shown in Figure 3 a (black and yellow curves, respectively). In particular, the current flowing through devices based on Apo-Azurins is significantly lower, due to the absence of a metal atom capable of mediating electron transfer, according to previous reports on metalloproteins and their apo-forms. ${ }^{[11,19]}$

To illustrate the fine structure in the transport data we plot the normalized derivative of the blue-copper Azurin data in Figure $3 \mathrm{~b}$. Here we can identify a series of peaks and valleys, in particular a pronounced double-peak structure at negative bias (e.g., a strong peak at $-2.3 \mathrm{~V}$ with a shoulder at $-2.2 \mathrm{~V}$ ) that shifts for different samples by some hundred millivolts. At positive bias we find broad peaks at $2.2 \mathrm{~V}, 2.8 \mathrm{~V}$, and $3.2 \mathrm{~V}$. These features were reproducible in different devices $(30 \%$ from an ensemble of 20 fabricated devices) and furthermore, no influence on $L_{\mathrm{y}}$ was observed.

We ascribe the multiple peaks in the $\mathrm{d} I / \mathrm{d} V$ curves to blue-copper proteins that participate in parallel to the current transport. This finding is supported by the almost symmetric position of the $\mathrm{d} I / \mathrm{d} V$ peaks with respect to the origin (all peaks are in the range $\pm 2.2 \mathrm{~V}$ to $\pm 3.4 \mathrm{~V}$ ). The specific potential drop across each protein, and therefore the effective potential of the redox centers, is expected to change for the different proteins immobilized inside the nanogap due to irregularities in the contacts and the different "lever arm" to the redox center. This leads to small differences in the resonant transport conditions for each immobilized protein.

NDR has been associated with 1) transfer of carriers from a high-mobility to a low-mobility valley, ${ }^{[20]} 2$ ) resonant tunneling between localized states, ${ }^{[21,22]}$ and 3) two-step reduction processes in the molecules. ${ }^{[23,24]}$ However, despite the current understanding of NDR in conventional semiconductors, this phenomenon is less understood on the molecular scale (see a previous report ${ }^{[13]}$ ). We propose the occurrence of on/off resonance conditions in the tunneling process through the redox levels (RSSR and $\mathrm{Cu}^{\mathrm{II}}$ ) of Azurin as the governing mechanism for current transport in our devices. Electron-transfer reactions in proteins in aqueous solutions have been investigated in considerable detail in the last years because of their central role in a number of biological processes, ranging from respiration to photosynthesis and energy conversion. To date, experimental information about this phenomenon has been provided primarily by spectroscopic and electrochemical techniques that measure various quantities averaged over a large number of molecules. It is commonly accepted that long-range ET in proteins occurs between a donor (D) and an acceptor (A) via vibration-induced electron tunneling, ${ }^{[25]}$ with a rate given by

$k=\frac{(2 \pi)^{2}}{h} H_{\mathrm{DA}}^{2} F C$

Here, $F C$ is the nuclear (Franck-Condon) factor, which in the Marcus model is approximately proportional to $\exp \left(-\Delta G^{*} / k_{\mathrm{B}} T\right)$. The activation energy, $\Delta G^{*}=\frac{\lambda}{4}\left(1-\frac{\Delta G^{0}}{\lambda}\right)^{2}$, in turn is determined by the "reorganization energy", $\lambda$, and the free energy of the reaction, $-\Delta G^{0}{ }^{[26]}$ On the other hand, $H_{\mathrm{DA}}^{2}=C \exp (-\beta R)$ is the tunneling matrix element from donor to acceptor, primarily depending on the distance $R$ between $\mathrm{D}$ and $\mathrm{A}(\beta \cong 1.1 \AA$ from Azurin protein studies $\left.{ }^{[27]}\right)$. The pre-exponential term $C$ depends on the character of the coupling between $\mathrm{D}$ and $\mathrm{A}$, and on the microenvironment separating $\mathrm{D}$ and $\mathrm{A}$, which mediates the virtual state or provides intermediate states (if $\mathrm{D}$ and $\mathrm{A}$ are in contact $H_{\mathrm{DA}}$ is roughly proportional to the overlap between their orbitals). An intriguing issue in understanding ET in proteins is to identify which part of the protein environment plays a dominant role in the electron tunneling between the redox pair ( $\mathrm{D}$ and $\mathrm{A}$ ), that is, the electron-tunneling pathway. ${ }^{[28]}$ 
In the case of Azurin, its $\beta$-barrel structure contains two potential redox centers: a blue-copper site coordinated directly to amino acid residues, and a disulfide bridge (Cys3Cys26) at the opposite end of the molecule, at a distance of $\approx 2.6 \mathrm{~nm}$ from the copper site. ${ }^{[2]}$ Intramolecular electron transfer from the disulfide radical ion $\mathrm{RSSR}^{-}$to the $\mathrm{Cu}^{\mathrm{II}}$ center was reported by Farver et al. ${ }^{[30]}$ who also identified the amino acids that provide the pathway with the strongest coupling (Figure $3 \mathrm{c}$ ). Our results are consistent with transport via this electron pathway connecting the two different redox-active sites in Azurin (namely the $\mathrm{Cu}^{\mathrm{II}}$ center and the disulfide bridge RSSR linking cysteines-3 and -26). In this frame, NDR can be attributed to resonant tunneling resulting from the alignment of the two narrow energy states localized on $\mathrm{Cu}^{\mathrm{II}}$ and in the region of RSSR (Cys3-Cys26), ${ }^{[31]}$ which leads to the maxima in the $I-V$ characteristics. A quantitative comparison between the ET rate obtained here and those quoted in the literature, provided primarily by spectroscopic and electrochemical techniques, is not possible because of the different environmental conditions. As an illustration of this, the ET rate constants determined by $\mathrm{AC}$ impedance and electroreflectance (ER) techniques $\left(300 \mathrm{~s}^{-1}\right.$ and $150-200 \mathrm{~s}^{-1}$, respectively) are one to two orders of magnitude higher than those determined by cyclic voltammetry $\left(4-12 \mathrm{~s}^{-1}\right) \cdot{ }^{[32]}$ Another important difference is that the reorganization energy in the molecular tunnel junction ${ }^{[33]}$ is significantly smaller than in the semi-infinite solvent space of electrochemical $\mathrm{ET}^{[2]}$ since in solution the dominant contribution to $\lambda\left(1.0 \mathrm{eV}\right.$ in a previous report $\left.{ }^{[29]}\right)$ arises from reorientation of solvent molecules in response to the change in charge distribution of the reactants. ${ }^{[34]}$ Finally, we note that in Farver's experiment, the created disulfide radical ion decays spontaneously by an intramolecular ET to the $\mathrm{Cu}^{\text {II }}$ center, while in our experiment an intense electric field is present in the tunneling junction, which can strongly enhance this process. Notably, our results are in good agreement (for both current values and peak position ranges) with the signatures of resonant tunneling (and NDR) recently reported in the current-voltage characteristics of a $\mathrm{Cu}$-Azurin metalloprotein, measured with conducting-probe atomic force microscopy junctions at low applied forces at Oxford University. ${ }^{[13,35]}$ However, in that report the mechanism and the protein sites responsible for the NDR were not identified. Since this latter work employed quite similar experimental conditions with respect to ours (with electron transfer reactions of few/individual molecules probed in high electric fields) the mechanism that we propose to explain NDR is also valid for their case. ${ }^{[36]}$

Finally, we consider the broadness of the peaks measured in the $\mathrm{d} I / \mathrm{d} V$ spectra. The sharpness of a resonant tunneling peak is related to the width of the involved levels/ bands, which is broadened by the interaction with the environment. In this respect, the metal redox centers provide low-lying highest-occupied molecular orbital (HOMO) and lowest-unoccupied molecular orbital (LUMO) levels that are strongly coupled to the vibrational nuclear environment. We attribute the observed broad peaks to fluctuations in the nuclear configuration, which shift the energy levels of the redox centers. ${ }^{[33,37]}$
In summary, we have 1) significantly improved the mesa-gap technique for the fabrication of large-scale arrays of nanojunctions/nanodevices, and 2) investigated transport in molecular tunnel junctions based on the blue-copper protein Azurin under ambient conditions, for the first time at the single-molecule level in device geometry. On the one hand, these results were achieved by our novel fabrication approach, which has a great appeal for applications in molecular electronics (especially for low-cost mass production of single-molecule components working at ambient conditions). On the other hand, we observed characteristic molecular features, such as NDR, in the $I-V$ spectra providing information on the pathway and the underlying mechanisms governing tunneling through the Azurin redox levels. These results are also very interesting from a technological point of view because of the possible applications in biosensors and switchable devices (exploiting NDR).

\section{Keywords:}

arrays $\cdot$ charge transport - molecular electronics •

nanotechnology $\cdot$ tunnel junctions

[1] G. Maruccio, R. Cingolani, R. Rinaldi, J. Mater. Chem. 2004, 14, 542.

[2] J. D. Zhang, M. Grubb, A. G. Hansen, A. M. Kuznetsov, A. Boisen, H. Wackerbarth, J. Ulstrup, J. Phys. Condens. Matter 2003, 15, S1873 and references therein.

[3] Q. J. Chi, J. D. Zhang, J. U. Nielsen, E. P. Friis, I. Chorkendorff, G. W. Canters, J. E. T. Andersen, J. Ulstrup, J. Am. Chem. Soc. 2000, 122, 4047.

[4] J. W. Zhao, J. J. Davis, M. S. P. Sansom, A. Hung, J. Am. Chem. Soc. 2004, 126, 5601.

[5] M. D. Austin, H. X. Ge, W. Wu, M. T. Li, Z. N. Yu, D. Wasserman, S. A. Lyon, S. Y. Chou, Appl. Phys. Lett. 2004, 84, 5299.

[6] G. Maruccio, P. Visconti, V. Arima, S. D’Amico, A. Blasco, E. D’Amone, R. Cingolani, R. Rinaldi, S. Masiero, T. Giorgi, G. Gottarelli, Nano Lett. 2003, 3, 479.

[7] S. J. Tans, A. R. M. Verschueren, C. Dekker, Nature 1998, 393, 49.

[8] J. Park, A. N. Pasupathy, J. I. Goldsmith, C. Chang, Y. Yaish, J. R. Petta, M. Rinkoski, J. P. Sethna, H. D. Abruna, P. L. McEuen, D. C. Ralph, Nature 2002, 417, 722.

[9] A. Bachtold, P. Hadley, T. Nakanishi, C. Dekker, Science 2001, 294, 1317.

[10] Y. Huang, X. F. Duan, Y. Cui, L. J. Lauhon, K. H. Kim, C. M. Lieber, Science 2001, 294, 1313.

[11] G. Maruccio, A. Biasco, P. Visconti, A. Bramanti, P. P. Pompa, F. Calabi, R. Cingolani, R. Rinaldi, S. Corni, R. Di Felice, E. Molinari, M. R. Verbeet, G. W. Canters, Adv. Mater. 2005, 17, 816.

[12] R. Krahne, A. Yacoby, H. Shtrikman, I. Bar-Joseph, T. Dadosh, J. Sperling, Appl. Phys. Lett. 2002, 81, 730.

[13] J. J. Davis, N. Wang, A. Morgan, T. T. Zhang, J. W. Zhao, Faraday Discuss. 2006, 131, 167.

[14] E. Vijgenboom, J. E. Busch, G.W. Canters, Microbiology 1997, $143,2853$.

[15] G. Gilardi, T. Den Blaauwen, G. W. Canters, J. Controlled Release 1998, 29, 231.

[16] Of course, proteins can attach to both electrodes. So, two configurations are possible.

[17] We fabricated 20 devices and observed the NDR in $30 \%$ of them. 


\section{communications}

[18] P. P. Pompa, A. Bramanti, G. Maruccio, L. L. del Mercato, R. Cingolani, R. Rinaldi, Chem. Phys. Lett. 2005, 404, 59.

[19] D. G. Xu, G. D. Watt, J. N. Harb, R. C. Davis, Nano Lett. 2005, 5 , 571.

[20] L. L. Chang, L. Esaki, R. Tsu, Appl. Phys. Lett. 1974, 24, 593.

[21] A. Patané, A. Polimeni, L. Eaves, P. C. Main, M. Henini, A. E. Belyaev, Y. V. Dubrovskii, P. N. Brounkov, E. E. Vdovin, Y. N. Khanin, G. Hill, Phys. Rev. B 2000, 62, 13595.

[22] N. P. Guisinger, M. E. Greene, R. Basu, A. S. Baluch, M. C. Hersam, Nano Lett. 2004, 4, 55.

[23] J. Chen, M. A. Reed, A. M. Rawlett, J. M. Tour, Science 1999, 286, 1550.

[24] J. M. Seminario, A. G. Zacarias, J. M. Tour, J. Am. Chem. Soc. 2000, 122, 3015.

[25] A. Broo, S. Larsson, J. Phys. Chem. 1991, 95, 4925.

[26] S. Larsson, A. Broo, L. Sjolin, J. Phys. Chem. 1995, 99, 4860.

[27] R. Ramamurthy, V. Ramamurthy, Organic and Inorganic Photochemistry, Marcel Dekker Inc., New York, 1998.

[28] In the pathway model suggested by Beratan and Onuchic, specific electron tunneling pathways exist in which chemical bonds are predominant, hydrogen bonds are subsidiary, and throughspace routes are rare.

[29] O. Farver, Y. Lu, M. C. Ang, I. Pecht, Proc. Natl. Acad. Sci. USA 1999, 96, 899.

[30] O. Farver, I. Pecht, J. Am. Chem. Soc. 1992, 114, 5764.

[31] The disulfide bonds are supposed to break during bonding. However, in their work, Farver et al. reported intramolecular electron transfer from the disulfide radical ion $\mathrm{RSSR}^{-}$to the $\mathrm{Cu}^{\prime \prime}$ and it is known that the reduction of a disulfide is dissociative and leads to the cleavage of the $\mathrm{S}-\mathrm{S}$ bond. As a consequence, our analogy with the Farver et al. case is still valid and we can continue to refer to their electron-tunneling pathway (we believe that a localized redox state located in the region of RSSR exists under the described experimental conditions).

[32] J. F. Rusling, Biomolecular Films: Design, Function, and Applications, Marcel Dekker Inc., New York, 2003.

[33] E. P. Friis, Y. I. Kharkats, A. M. Kuznetsov, J. Ulstrup, J. Phys. Chem. A 1998, 102, 7851.

[34] H. B. Gray, J. R. Winkler, Annu. Rev. Biochem. 1996, 65, 537.

[35] J. J. Davis, D. A. Morgan, C. L. Wrathmell, D. N. Axford, J. Zhao, N. Wang, J. Mater. Chem. 2005, 15, 2160.

[36] For the sake of completeness, despite the many similarities we should mention also the differences in the experimental conditions between our work and that of the group at Oxford University concerning the applied forces that can result in difficulties in interconnecting a single protein at low forces, while higher forces can destroy this fragile molecule. This would explain also why NDR is visible only in a narrow window in the Oxford experiments.

[37] N. J. Tao, Phys. Rev. Lett. 1996, 76, 4066.

Received: October 28, 2006

Revised: April 1, 2007

Published online on May 18, 2007 